\title{
Clinical and Economic Burden of Patients with Chronic Hepatitis $C$ with Versus Without Antiviral Treatment in Japan: An Observational Cohort Study Using Hospital Claims Data
}

\author{
Kiyotaka Yamazaki · Dendy Macaulay · Yan Song · Yuri Sanchez Gonzalez
}

Received: December 12, 2018 / Published online: February 15, 2019

(C) The Author(s) 2019

\begin{abstract}
Introduction: Japan has one of the highest prevalence rate of chronic hepatitis $\mathrm{C}(\mathrm{CHC})$ in the industrialized world. However, the burden of CHC treatment is poorly understood. Thus, the healthcare resource utilization and costs of treated versus untreated patients, and patients with early versus delayed treatment initiation, were assessed in Japan.

Methods: Adult patients with $\geq 2$ CHC diagnoses were identified from the Medical Data Vision hospital claims database (1 April 2008-31 May 2016). The presence or absence of antiviral treatment claims was used to form the treated and untreated cohorts, respectively. Among treated patients, the presence of a cirrhosis-related diagnosis was used as an indicator of delayed treatment. The index date was
\end{abstract}

Enhanced digital features To view enhanced digital features for this article go to https://doi.org/10.6084/ m9.figshare.7628900.

K. Yamazaki

AbbVie GK, Tokyo, Japan

D. Macaulay

Analysis Group Inc., New York, NY, USA

Y. Song

Analysis Group Inc., Boston, MA, USA

Y. Sanchez Gonzalez $(\bowtie)$

AbbVie Inc., North Chicago, IL, USA

e-mail: Yuri.SanchezGonzalez@AbbVie.com defined as the date of the first antiviral claim for treated patients and randomized to any date with a medical visit for untreated patients. Annualized total healthcare costs and costs associated with hepatic manifestations (HMs) or extrahepatic manifestations (EHMs) were evaluated from the index date to the last observed medical visit.

Results: Of 100,125 patients with CHC, 12,984 were treated (early: 8104, delayed: 4880) and 87,141 were untreated. After adjusting for covariates, untreated patients had $¥ 613,034$ (\$5456 USD; $¥ 1=\$ 0.0089$ ) higher annual medical costs compared with treated patients $(P<0.001)$, a difference driven by higher inpatient costs. Between 65\% (treated patients) and $70 \%$ (untreated patients) of medical costs were EHM-related and between 14\% (untreated patients) and 15\% (treated patients) were HMrelated. Patients in the delayed treatment cohort had $¥ 114,347$ (\$1018) higher annual medical costs $(P<0.001)$ versus those in the early treatment cohort. About $95 \%$ of these costs were EHM-related, and 64\% were HMrelated.

Conclusion: Withholding or delaying antiviral treatment initiation for Japanese patients with $\mathrm{CHC}$ increases the clinical and economic burden associated with HMs and EHMs.

Funding: AbbVie. 
Keywords: Costs; Direct acting antivirals; Healthcare resource utilization; Hepatitis C; Real world

\section{INTRODUCTION}

Hepatitis $\mathrm{C}$ is an infectious liver disease caused by hepatitis $\mathrm{C}$ virus $(\mathrm{HCV})[1,2]$ that progresses to a chronic illness [chronic hepatitis $\mathrm{C}(\mathrm{CHC})]$ in $50-80 \%$ of patients. CHC entails a high risk of debilitating liver-related complications such as cirrhosis, liver failure, and hepatocellular carcinoma (HCC) [1, 2]. Moreover, other organs can be impacted, leading to extra-hepatic manifestations (EHMs) that also contribute to the disease burden $[3,4]$. As a result of historical events $[5,6]$, Japan is the country with the highest prevalence of $\mathrm{CHC}$ in the industrialized world, with $1.5 \%$ of its population infected compared with a worldwide prevalence of $1.1 \%$ $[5,7,8]$. The prevalence of CHC in Japan steadily increases with age [9]; hence, this country has the oldest HCV-infected population [2].

The treatment landscape of $\mathrm{CHC}$ has drastically changed with the approval of interferon (IFN)-free direct-acting antivirals (DAAs). These treatments have demonstrated substantial improvements in infection clearance rates relative to interferon-based therapies [1, 10-12]. Despite their high efficacy, several real-world studies have shown that only a minority of patients with CHC initiate therapy because of treatment ineligibility (e.g., advanced comorbidities), patient refusal, physician's decision to defer treatment, or denial by the healthcare insurer [13-16]. Thus, there may be a substantial burden associated with withholding or deferring CHC therapy, but data on this research question are limited in Japan [17]. Furthermore, there is a dearth of information on the clinical and economic burden of EHMs and hepatic manifestations (HMs). This study aims to fill these knowledge gaps by evaluating and comparing the costs and healthcare resource utilization (HRU) of Japanese patients with CHC between (1) treated versus untreated patients and (2) patients with early versus delayed CHC treatment initiation.

\section{METHODS}

\section{Data Source}

Data from the Medical Data Vision (MDV) hospital claims database (1 April 2008-31 May 2016) were analyzed. The MDV database contains patient medical records from 247 acute care hospitals, representing approximately $16 \%$ of all acute care hospitals in Japan. The database contains disease diagnoses, claims for medical procedures and pharmacy prescriptions (from inpatient and outpatient services in the hospitals covered), and laboratory test results (available for $\sim 10 \%$ of patients). This article is based on previously available and de-identified data and does not involve any new studies of human or animal subjects performed by any of the authors. Appropriate permission from Medical Data Vision (MDV), the data provider, was obtained before using and analyzing the data.

\section{Study Population}

Eligible patients were required to be $\geq 18$ years of age and to have received $\geq 2$ diagnoses of CHC (International Classification of Diseases, 10th revision code: B18.2) on distinct dates. The use of two diagnoses as an inclusion criterion aims to mitigate the risk associated with potential coding errors [18-20].

Patients were assigned to the treated or untreated cohort based on the presence or absence of claims for $\mathrm{CHC}$ antiviral treatments available in Japan at the time of data analysis. These included both IFN-based and IFN-free treatments prescribed after the first observed diagnosis of CHC. For patients in the treated cohort, the index date was defined as the date of the first claim for an antiviral treatment. For patients in the untreated cohort, the index date was randomly selected among dates on which a medical service was performed in an inpatient or outpatient setting after the first observed diagnosis of $\mathrm{CHC}$. The baseline period was defined as the first 6 months prior to the index date. To ensure that patients had been observed since the baseline period, patients were required to have received $\geq 1$ medical service before the 
baseline period (but within 2 years prior to the index date).

Treated patients were assigned to the delayed treatment cohort or the early treatment cohort based on liver disease stage. A diagnosis of cirrhosis or a related condition [21] any time before treatment initiation or within 6 months after treatment initiation was used to classify patients in the delayed treatment cohort, and the remaining patients were assigned to the early treatment cohort. The study period was defined as the period spanning from the index date to the last observed medical visit.

\section{Baseline Characteristics}

Patient characteristics were measured during the baseline period and included the following categories: demographics, disease characteristics, comorbidity profile, HRU and associated costs, EHMs, and HMs [3]. EHMs included cardiovascular disease, metabolic conditions, malignancy, kidney disease, neuro-muscular manifestations, autoimmune disease, and others (cognitive impairment, depression, gastroesophageal reflux disease, and irritable bowel syndrome). HMs included cirrhosis and related complications (esophageal varices, spontaneous bacterial peritonitis, hepatic encephalopathy, portal hypertension, ascites, splenomegaly, hepatorenal syndrome, HCC, and porphyria cutanea tarda) and liver transplantation. All evaluated characteristics within each of these categories are listed in Table 1.

\section{Study Outcomes}

The following aspects of HRU were evaluated during the study period: all-cause, EHM-related, and HM-related inpatient and outpatient visits; HCV-related laboratory tests; liver cancer marker tests; diagnostic imaging; and liver biopsies. EHM- or HM-related HRU was defined as a medical service associated with a diagnosis of an EHM or HM, respectively.

Medical service costs and pharmacy costs were summarized for each cohort and included all-cause, EHM-related, and HM-related inpatient or outpatient visit costs. Pharmacy costs were stratified into $\mathrm{CHC}$ - and non-CHC-related costs, with CHC-related costs defined as pharmacy costs associated with CHC treatments. Japanese yen (¥) was converted to US dollars (\$) using the exchange rate of $\$ 1=¥ 112$ (as of 20 November 2017) [22].

\section{Statistical Analyses}

Means and standard deviations were used to summarize continuous variables; frequencies and percentages were used to summarize categorical variables. Patient characteristics between study cohorts were compared using Wilcoxon rank-sum tests for continuous variables and chisquare tests for categorical variables.

Incidence rates (IRs) were described for each HRU outcome in each cohort. Unadjusted and adjusted incidence rate ratios (IRRs), including 95\% confidence intervals (CIs) and $P$ values, were calculated using generalized linear models with a log link and Poisson distribution. The adjusted models controlled for baseline covariates, including age, sex, index year, time from initial CHC diagnosis, cirrhosis, HCC, Quan-Charlson comorbidity index (a score that predicts in-hospital mortality over a 1-year period based on the presence of Charlson comorbidities [23, 24]), cardiovascular disease, and metabolic conditions.

The unit cost for each medical procedure and prescription was obtained from the 2016 Japanese National Health Insurance Fee Schedule [25]. All medical service and pharmacy costs were calculated for each patient and annualized for patients with $>1$ day of follow-up. Costs were compared between cohorts using generalized linear models with a log link and Tweedie distribution. Adjusted models controlled for the same covariates as in the models of IRRs. Both HRU and cost regressions included indicator variables for either the treated cohort or delayed treatment as key explanatory variables to test study hypotheses.

\section{RESULTS}

\section{Baseline Characteristics}

A total of 100,125 adult patients with $\mathrm{CHC}$ were identified, including 12,984 treated and 87,141 


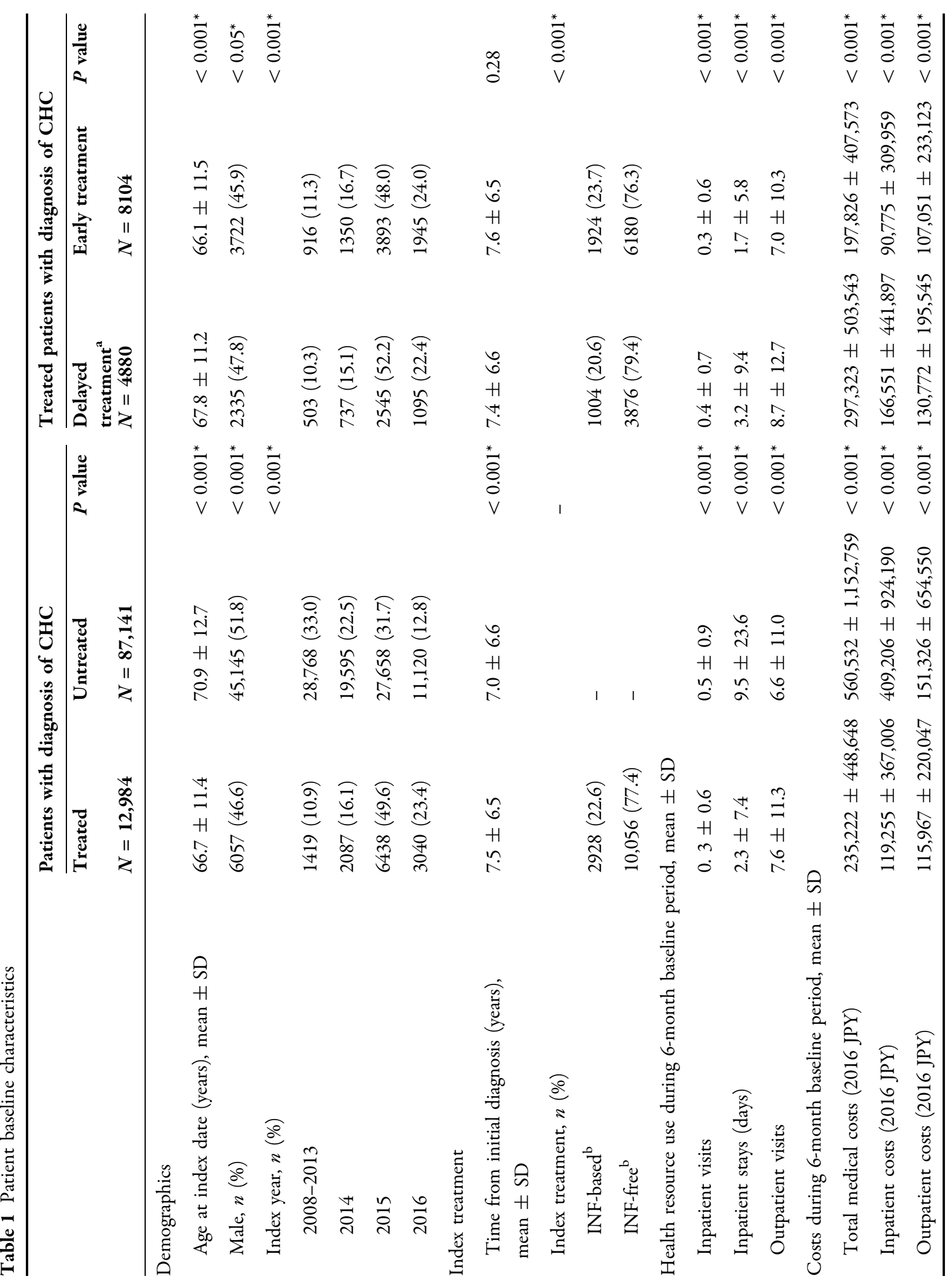




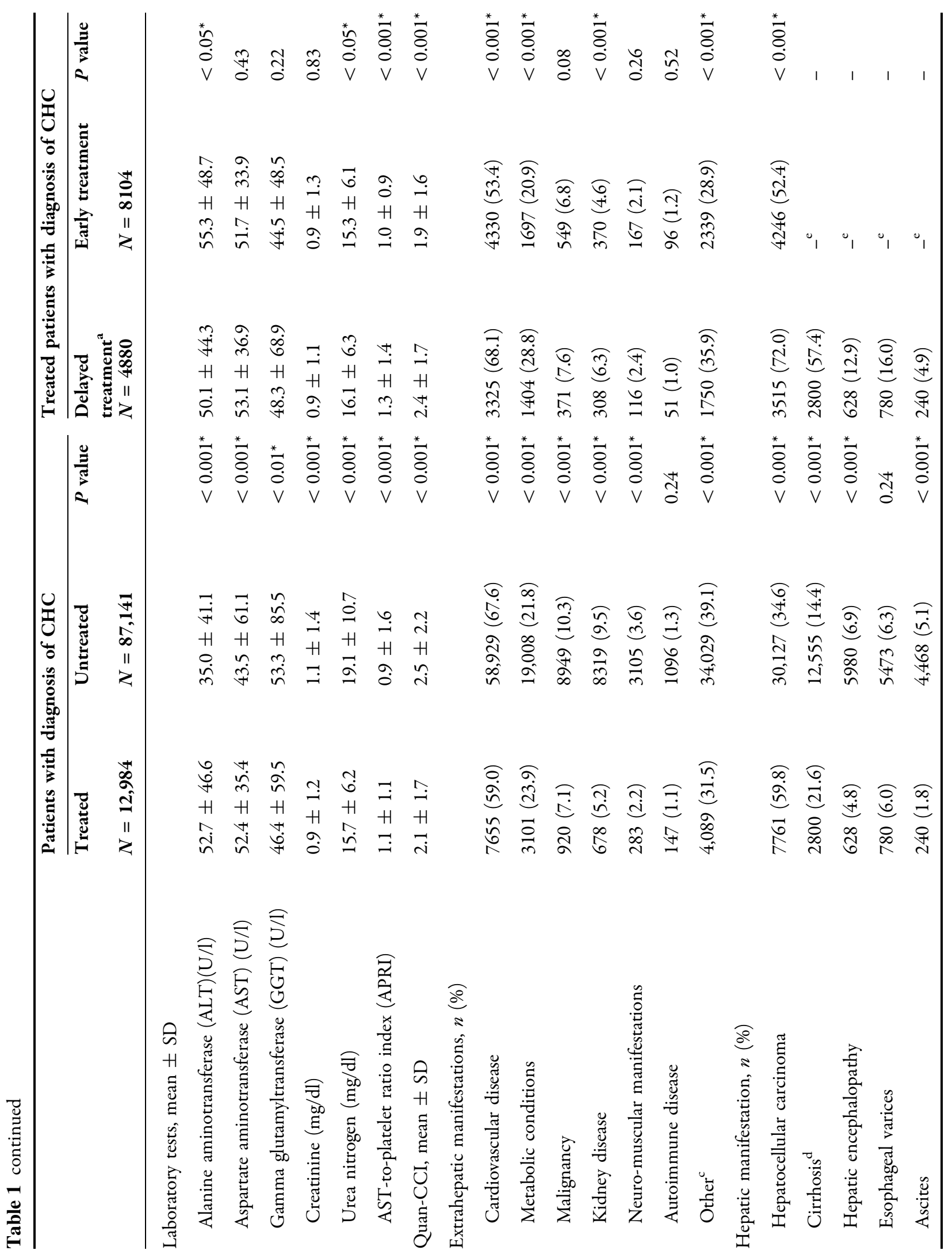




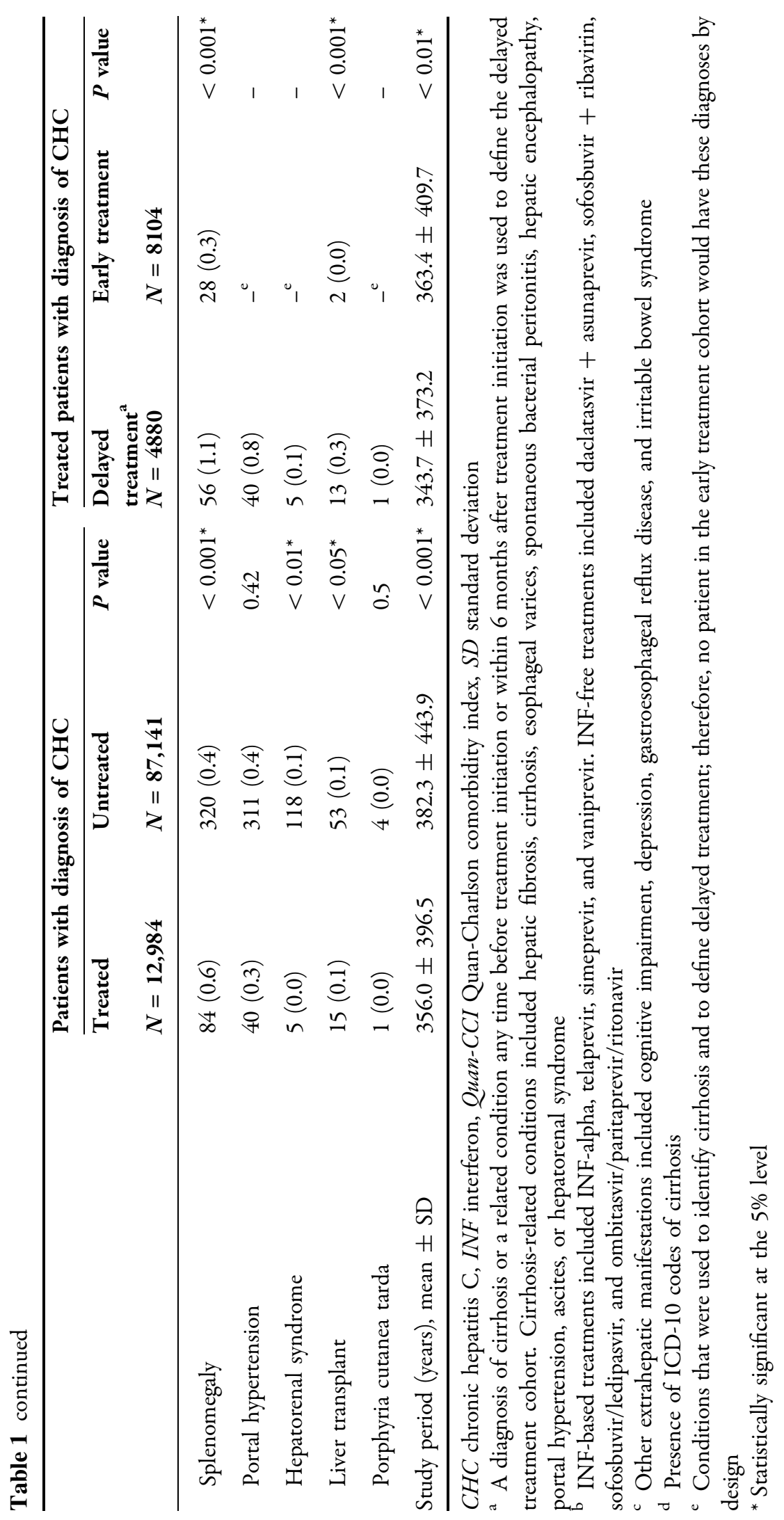




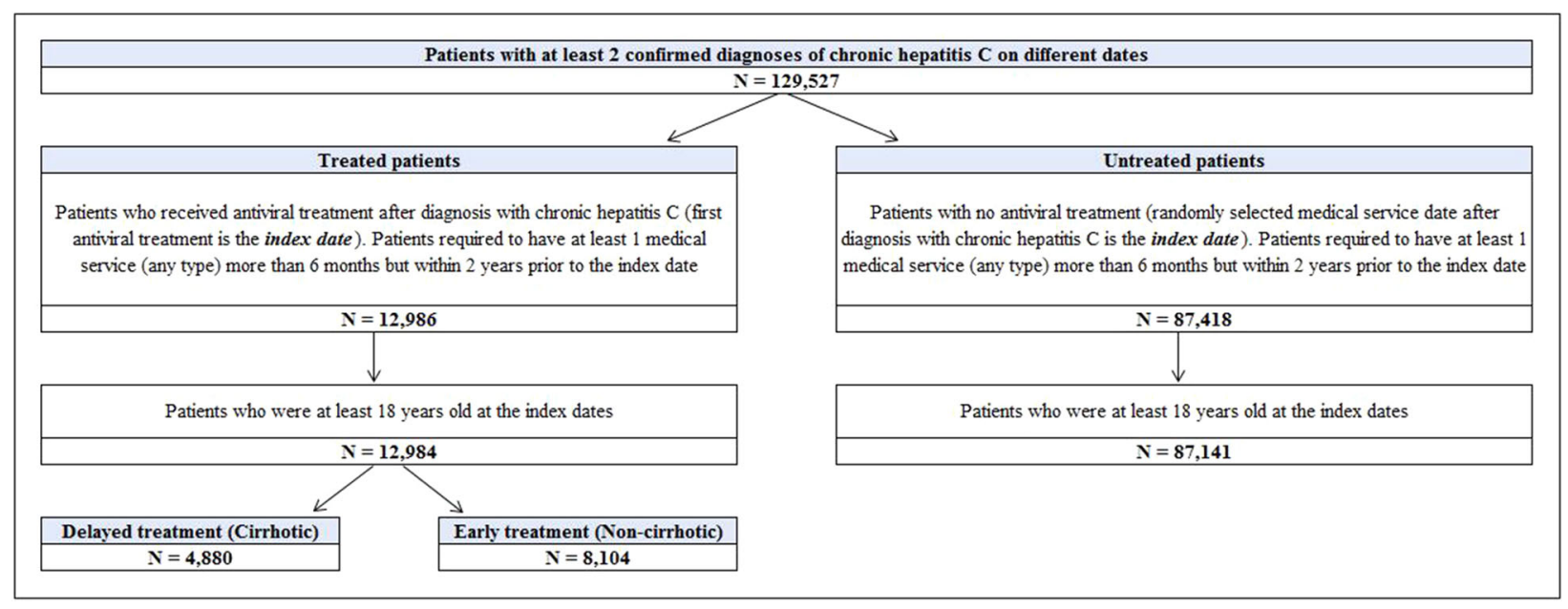

Fig. 1 Sample selection flow

untreated patients (Fig. 1). Compared with untreated patients, treated patients were younger, included a lower proportion of males, and had a longer disease duration since initial CHC diagnosis (all $P<0.001$; Table 1).

Among treated patients, $89 \%$ received their treatment from 2014 onwards (the year of approval of the first IFN-free DAA in Japan), and $77 \%$ received an IFN-free treatment (Table 1 ). On average, treated patients had fewer inpatient days and lower medical service costs during baseline, but more outpatient visits (all $P<0.001)$. For most evaluated EHMs at baseline, the proportion of affected patients was lower among treated patients relative to untreated patients, while a higher proportion of treated patients had HMs such as HCC (60\% vs. $35 \%, P<0.001)$ and cirrhosis $(22 \%$ vs. $14 \%$, $P<0.001)$. This suggests treatment was restricted or prioritized to patients with the presence or risk of advanced liver disease.

Among CHC patients who received antiviral treatments, 4880 and 8104 had a delayed and early treatment initiation, respectively (Fig. 1). Statistically significant differences were observed in terms of age and gender between the delayed and early treatment cohorts (both $P<0.001$ ), and disease duration since initial diagnosis was similar between both cohorts. Patients in the delayed treatment cohort had higher baseline HRU and costs compared with patients in the early treatment cohort (both
$P<0.001)$. The proportion of patients diagnosed with HCC prior to antiviral treatment initiation was higher among patients in the delayed treatment cohort compared with patients in the early treatment cohort ( $72 \%$ vs. $52 \%, P<0.001)$. In addition, the proportions of patients with EHMs were higher among patients in the delayed treatment cohort compared with the early treatment cohort.

\section{Healthcare Resource Uutilization}

The average durations of follow-up were 356 and 382 days for treated and untreated patients, respectively. The results showed that the treatment of CHC could partially shift the burden of HRU from an inpatient to an outpatient setting. Relative to patients in the untreated cohort, IRs of all-cause, EHM-related, and HM-related inpatient visits were significantly lower among treated patients (adjusted IRRs: 0.67, 0.65, and 0.58, respectively, all $P<0.001$; Table 2). Patients in the treated cohort had higher IRs of all-cause, EHM-related, and HM-related outpatient visits relative to those in the untreated cohort (adjusted IRR: 1.59, 1.44, and 1.27, respectively, all $P<0.001)$. Disease-monitoring tests, including HCV-related laboratory tests, liver cancer marker tests, and diagnostic imaging, were performed more frequently among treated than untreated patients (all $P<0.001$ ). 
Table 2 Incidence rate ratios (IRRs) of healthcare resource utilization between treated and untreated patients

\begin{tabular}{|c|c|c|c|c|c|c|c|c|}
\hline & \multicolumn{2}{|c|}{ Incidence rate } & \multirow{2}{*}{$\begin{array}{l}\text { Unadjusted } \\
\text { IRR }^{\mathbf{a}}\end{array}$} & \multirow[t]{2}{*}{$95 \% \mathrm{CI}$} & \multirow[t]{2}{*}{$P$ value } & \multirow{2}{*}{$\begin{array}{l}\text { Adjusted } \\
\text { IRR }^{\mathbf{a}, \mathbf{b}}\end{array}$} & \multirow[t]{2}{*}{$95 \% \mathrm{CI}$} & \multirow[t]{2}{*}{$P$ value } \\
\hline & $\begin{array}{l}\text { Treated } \\
N=12,984\end{array}$ & $\begin{array}{l}\text { Untreated } \\
N=87,141\end{array}$ & & & & & & \\
\hline IP visits & 0.53 & 0.72 & 0.74 & $(0.68,0.80)$ & $<0.001^{*}$ & 0.67 & $(0.62,0.72)$ & $<0.001^{*}$ \\
\hline EHM-related & 0.44 & 0.64 & 0.69 & $(0.64,0.75)$ & $<0.001^{*}$ & 0.65 & $(0.60,0.69)$ & $<0.001^{*}$ \\
\hline HM-related & 0.30 & 0.34 & 0.89 & $(0.80,0.99)$ & $<0.05^{*}$ & 0.58 & $(0.54,0.62)$ & $<0.001^{*}$ \\
\hline OP visits & 23.18 & 14.04 & 1.65 & $(1.61,1.69)$ & $<0.001^{*}$ & 1.59 & $(1.55,1.63)$ & $<0.001^{*}$ \\
\hline EHM-related & 17.04 & 11.93 & 1.43 & $(1.39,1.47)$ & $<0.001^{*}$ & 1.44 & $(1.40,1.48)$ & $<0.001^{*}$ \\
\hline HM-related & 9.13 & 4.70 & 1.94 & $(1.86,2.03)$ & $<0.001^{*}$ & 1.27 & $(1.23,1.32)$ & $<0.001^{*}$ \\
\hline $\begin{array}{l}\mathrm{HCV} \text {-related } \\
\text { lab tests }\end{array}$ & 15.28 & 7.14 & 2.14 & $(2.10,2.19)$ & $<0.001^{*}$ & 1.89 & $(1.85,1.93)$ & $<0.001^{*}$ \\
\hline $\begin{array}{l}\text { Liver cancer } \\
\text { marker tests }\end{array}$ & 5.07 & 1.46 & 3.47 & $(3.37,3.57)$ & $<0.001^{*}$ & 2.41 & $(2.34,2.47)$ & $<0.001^{*}$ \\
\hline $\begin{array}{r}\text { Diagnostic } \\
\text { imaging }^{\mathrm{e}}\end{array}$ & 2.43 & 2.32 & 1.05 & $(1.01,1.08)$ & $<0.05^{*}$ & 0.87 & $(0.84,0.90)$ & $<0.001^{*}$ \\
\hline Liver biopsy & 0.08 & 0.08 & 1.02 & $(0.90,1.16)$ & 0.76 & 0.97 & $(0.86,1.10)$ & 0.65 \\
\hline
\end{tabular}

$C H C$ chronic hepatitis $\mathrm{C}, C I$ confidence interval, $E H M$ extrahepatic manifestations, $H C V$ hepatitis $\mathrm{C}$ virus, $H M$ hepatic manifestation, $I P$ inpatient, $I R R$ incidence rate ratio, $O P$ outpatient

${ }^{a}$ IRR $<1$ indicates that the incidence of the event was lower in treated patients compared to untreated patients

b Adjusted IRRs were calculated using generalized linear models with a log link and a Poisson distribution. The models were adjusted for age, gender, index year, time from initial diagnosis, Quan-CCI, cardiovascular disease, metabolic conditions, hepatocellular carcinoma, and cirrhosis

c HCV-related laboratory tests included HCV antibody tests, HCV RNA tests, and liver function tests

${ }^{d}$ Liver cancer marker tests included tests on alpha-fetoprotein (AFP), APF-L3\%, and PIVKA-2

${ }^{e}$ Diagnostic imaging included computerized tomography (CT), magnetic resonance imaging (MRI), and ultrasonography of the liver

* Statistically significant at the 5\% level

Among treated patients, the average followup time was 344 and 363 days for patients in the delayed and early treatment cohorts, respectively. The results showed that early initiation of CHC treatment could alleviate the HRU burden of CHC. Relative to patients in the early treatment cohort, those in the delayed treatment cohort had higher IRs of all-cause, EHMrelated, and HM-related inpatient visits (adjusted IRR: $1.43,1.48$, and 2.52 , respectively all $P<0.001$; Table 3). The IR of HM-related outpatient visits was higher for patients in the delayed treatment cohort than that in the early treatment cohort (adjusted IRR: 2.33, $P<0.001)$. Although the IRs of EHM-related and all-cause outpatient visits were also higher among patients in the delayed treatment cohort, the magnitude of the association was smaller (adjusted IRR: 1.04 and 1.03, both $P<0.05$, respectively). Patients in the delayed treatment cohort were monitored with more HCV-related laboratory tests, cancer marker tests, and diagnostic imaging tests compared with patients in the early treatment cohort (all $P<0.001)$.

\section{Costs}

Treatment of CHC was associated with significant savings in medical service costs. Untreated 
Table 3 Healthcare resource utilization between patients in the delayed treatment cohort and patients in the early treatment cohort

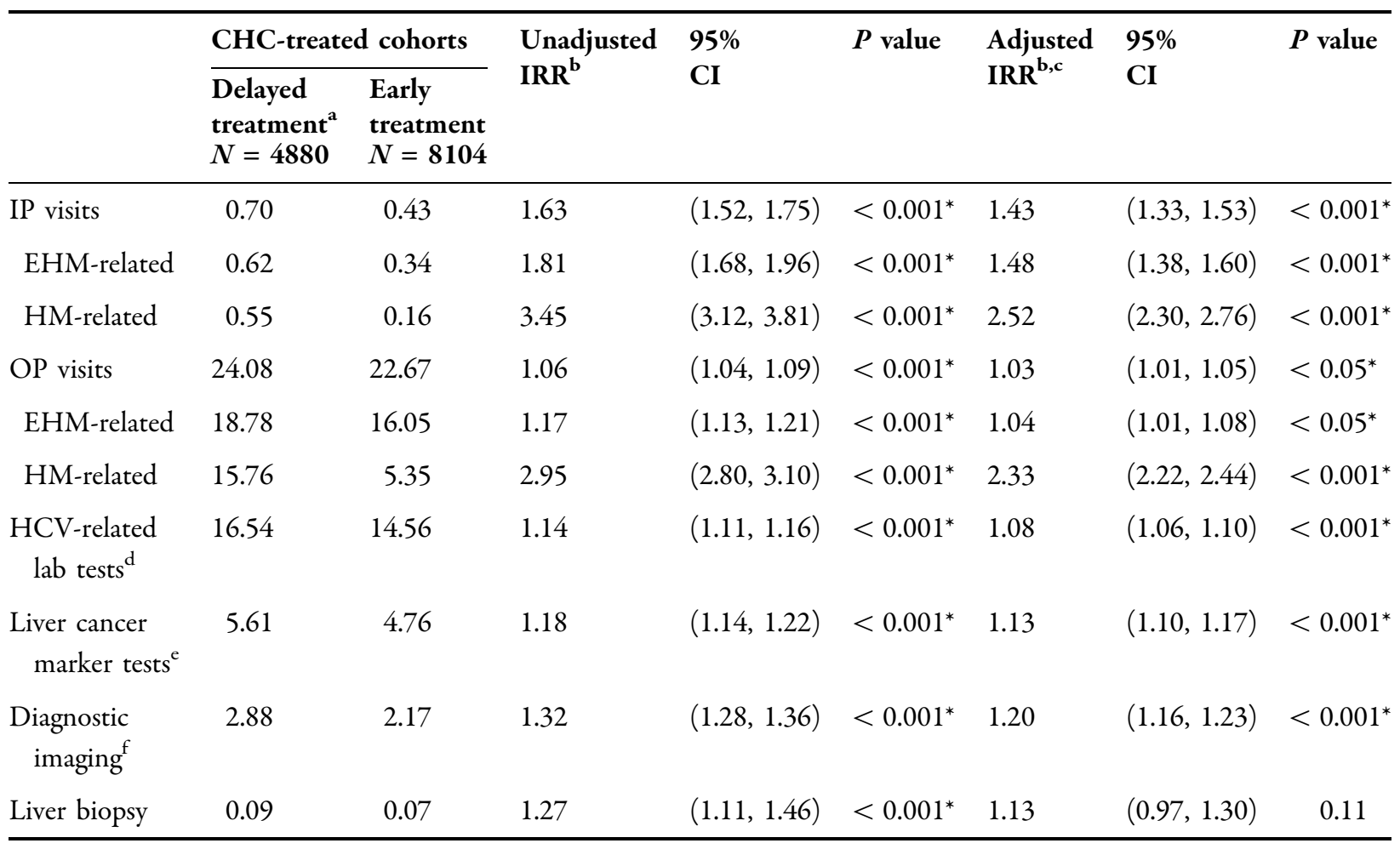

$C H C$ chronic hepatitis $\mathrm{C}, C I$ confidence interval, $E H M$ extrahepatic manifestations, $H C V$ hepatitis $\mathrm{C}$ virus, $H M$ hepatic manifestation, $I P$ inpatient, $I R R$ incidence rate ratio, $O P$ outpatient

${ }^{a}$ A diagnosis of cirrhosis or a related condition any time before treatment initiation or within 6 months after treatment initiation was used to define the delayed treatment cohort. Cirrhosis-related conditions included hepatic fibrosis, cirrhosis, esophageal varices, spontaneous bacterial peritonitis, hepatic encephalopathy, portal hypertension, ascites, or hepatorenal syndrome

${ }^{b}$ IRR $<1$ indicates that the incidence of the event was lower in patients with delayed treatment than in patients with early treatment

${ }^{c}$ Adjusted IRRs were calculated using generalized linear models with a log link and a Poisson distribution. The models were adjusted for age, gender, index year, time from initial diagnosis, Quan-CCI, cardiovascular disease, metabolic conditions, and hepatocellular carcinoma

d HCV-related laboratory tests included HCV antibody tests, HCV RNA tests, and liver function tests

${ }^{\mathrm{e}}$ Liver cancer marker tests included tests on alpha-fetoprotein (AFP), APF-L3\%, and PIVKA-2

${ }^{\mathrm{f}}$ Diagnostic imaging included computerized tomography (CT), magnetic resonance imaging (MRI), and ultrasonography of the liver

* Statistically significant at the $5 \%$ level

patients incurred $¥ 1,180,035(\$ 10,502)$ higher annual inpatient costs and $¥ 98,100$ (\$873) lower outpatient costs compared with treated patients, resulting in $¥ 1,081,935 \quad(\$ 9,629)$ higher total annual medical service costs (both $P<0.001$; Fig. 2). Between 79\% (treated patients) and $87 \%$ (untreated patients) of allcause costs were EHM-related, and 44\% (untreated patients) to $49 \%$ (treated patients) were HM-related. Similar results were observed after adjusting for baseline covariates [difference in annual medical service costs: $-¥ 613,034$ $(-\$ 5456) ; P<0.001]$.

While treatment of $\mathrm{CHC}$ entails high $\mathrm{CHC}$ related pharmacy costs, it can reduce pharmacy costs for non-CHC-related conditions. Patients 

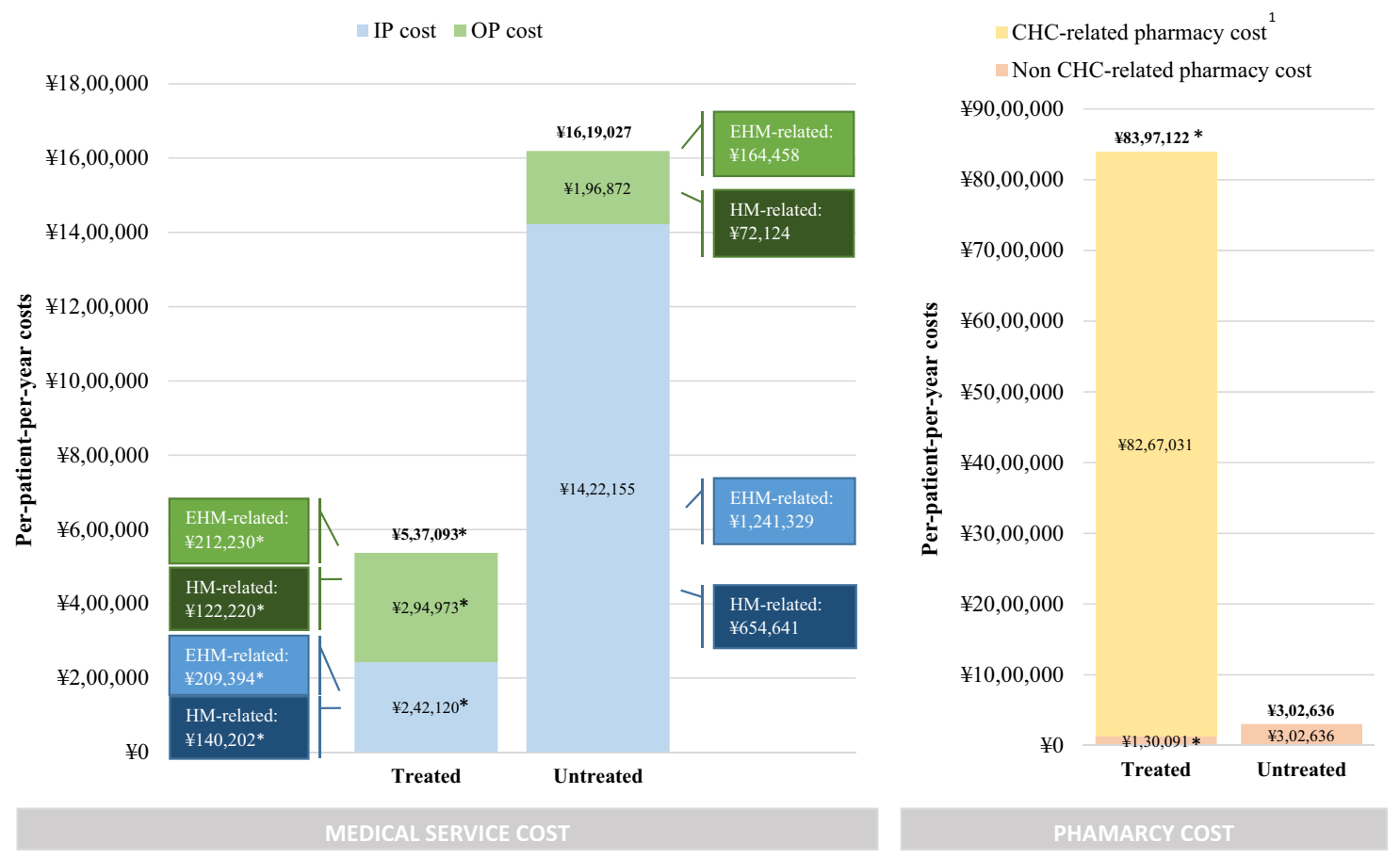

Fig. 2 Annual healthcare costs between treated and untreated patients stratified into medical service costs (left panel) and pharmacy costs (right panel). $C H C$ chronic hepatitis C, $E H M$ extrahepatic manifestations, $H M$ hepatic manifestations, $I P$ inpatient, $O P$ outpatient. ${ }^{*} P<0.05$ of the adjusted cost difference of treated versus untreated patients. $\$ 1$ USD $=¥ 112$ Japanese yen $(¥ 1=\$ 0.0089)$.

in the treated cohort had $¥ 8,094,485(\$ 72,042)$ higher all-cause pharmacy costs compared with patients in the untreated cohort during the study period (Fig. 2). In the treated cohort, 98\% of pharmacy costs were CHC-related, whereas non-CHC related pharmacy costs were $¥ 172,546$ (\$1536) lower compared with untreated patients $(P<0.001)$. Similar results were obtained after adjusting for baseline covariates [difference in annual pharmacy costs: $¥ 6,915,739$ (\$61,550); $P<0.001]$.

Early treatment initiation was associated with significant healthcare savings in medical costs. Among patients who received antiviral treatments, those in the delayed treatment cohort had ¥183,541 (\$1634) higher annual allcause medical service costs during the study period, a difference driven by $¥ 161,067$ (\$1433) higher inpatient costs and $¥ 22,474$ (\$200) higher outpatient costs compared with patients
${ }^{1}$ Exchange rate was obtained from the Bank of Japan on 20 November 2017. Since the study population mostly consists of patients initiated on an IFN-free DAA regimen, a large part of pharmacy costs can be considered nonrecurrent because of the high cure rates that can be achieved with such regimens over a standard 12- or 24-week treatment course (as opposed to medical costs)

treated early (both $P<0.001 ;$ Fig. 3 ). While the difference in the proportion of all-cause costs attributable to EHM-related costs was $12 \%$ higher among patients in the delayed treatment cohort ( $85 \%$ vs. $73 \%$ ), it reached $45 \%$ for costs attributable to HMs (73\% vs. $28 \%)$. Similar results were found after adjusting for baseline covariates [difference in annual medical service costs: ¥114,347 (\$1018); $P<0.001]$.

Early treatment initiation was also associated with a significant reduction in non-CHC-related pharmacy costs. Patients in the delayed treatment cohort had $¥ 196,919$ (\$1753) lower allcause pharmacy cost relative to those in the early treatment cohort $[¥ 8,274,214(\$ 73,641)$ vs. $¥ 8,471,133$ (\$75,393); Fig. 3], but the difference did not reach statistical significance. For both cohorts, $>98 \%$ of total pharmacy costs were CHC-related. Relative to patients in the early treatment cohort, those in the delayed 

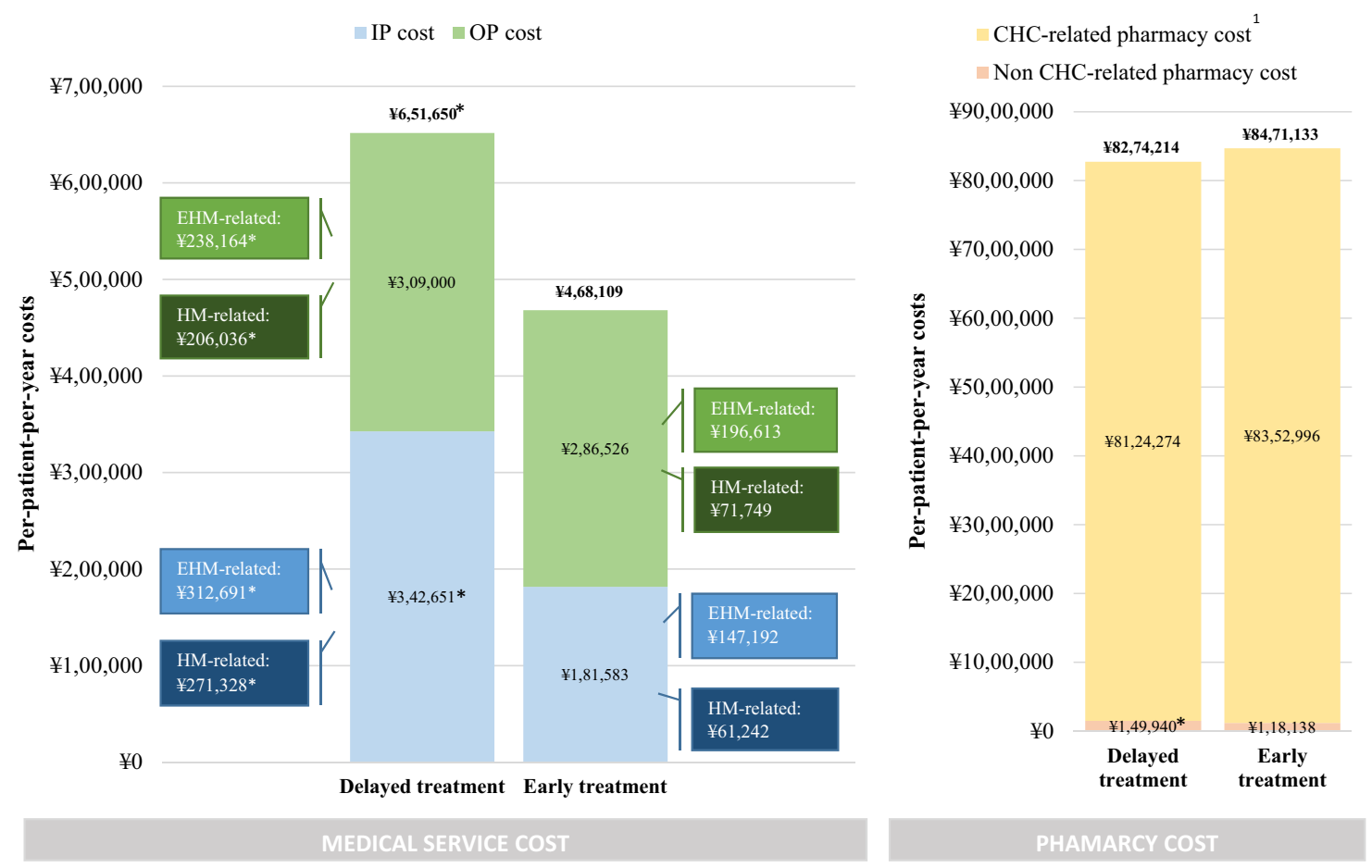

Fig. 3 Annual healthcare costs between patients in the delayed treatment cohort and patients in the early treatment cohort stratified into medical service costs (left panel) and pharmacy costs (right panel). CHC chronic hepatitis C, $E H M$ extrahepatic manifestations, $H M$ hepatic manifestations, $I P$ inpatient, $O P$ outpatient. ${ }^{*}$ Denotes $P<0.05$ of the adjusted cost difference of patients in the delayed treatment cohort versus patients in the early treatment cohort. $\$ 1$ USD $=¥ 112$ Japanese yen

treatment cohort had $¥ 31,802$ (\$283) higher non-CHC related pharmacy costs. Adjusted CHC-related pharmacy costs were similar between patients in the delayed and early treatment cohorts, while adjusted non-CHCrelated pharmacy costs remained significantly lower for patients in the early treatment cohort [¥23,079 (\$205); $P<0.001]$.

\section{DISCUSSION}

Results from this retrospective study suggest that the clinical and economic burden of $\mathrm{CHC}$ is substantial, but can be reduced with treatment. The annual medical costs among treated patients represented approximately half of those among untreated patients, and up to $75 \%$
(¥1=\$0.0089). ${ }^{1}$ Exchange rate was obtained from the Bank of Japan on 20 November 2017. Since the study population mostly consists of patients initiated on an IFNfree DAA regimen, a large part of pharmacy costs can be considered non-recurrent because of the high cure rates that can be achieved with such regimens over a standard 12- or 24-week treatment course (as opposed to medical costs)

of medical cost savings ensuing from $\mathrm{CHC}$ treatment were attributable to better management of EHMs. Early treatment initiation was associated with an even larger reduction in the clinical and economic burden of CHC. Together, these results highlight the incremental burden associated with untreated $\mathrm{CHC}$ and delayed treatment initiation and contribute to characterizing the population of $\mathrm{CHC}$ patients in Japan.

Treated patients with $\mathrm{CHC}$ were observed to have more outpatient visits and disease-monitoring laboratory tests compared with untreated patients, suggesting these patients may have been more actively monitored for disease progression. In addition to curing the underlying disease, this may help prevent the development of more severe HMs and EHMs as evidenced by 
the significantly lower rate of inpatient visits among treated patients. Consequently, treatment was associated with significantly lower medical service costs, which were driven by lower inpatient costs among treated versus untreated patients. Importantly, the adjusted annual savings in EHM-related inpatient costs of treated patients represented up to $84 \%$ of the total estimated benefit of treatment. This highlights that the economic impact of CHC symptoms extends beyond hepatic complications. Therefore, the higher pharmacy costs of CHC treatments, which are driven by one-time DAA treatment costs (i.e., non-recurrent fees due to the high cure rates achieved), are partially offset by an approximately $¥ 600,000$ (\$5340) reduction in adjusted medical service costs every year, which is driven by lower inpatient costs. With the one-time cost of DAAs ranging between $¥ 2.3$ (\$0.02) million and $¥ 4.6$ (\$0.04) million (estimated drug cost using the 2016 Japanese National Health Insurance Fee Schedule), the break-even horizon with the annual savings from medical services was 3.8-7.7 years. With recent and upcoming reductions in the prices of DAAs, this break-even horizon is expected to shorten, enhancing the economic rationale to remove restrictions to treatment (e.g., based on advanced age or poor prognosis).

In addition, early treatment initiation was associated with a significantly lower economic burden compared with delayed treatment initiation. As the most common baseline HM was HCC in both cohorts, this result suggests that a significant proportion of these savings may come from improved clinical prevention, monitoring, and management of HCC following early treatment initiation. In fact, LaMori et al. examined predictors of high HRU among patients with CHC [20]. The authors reported that the odds of belonging to the top $20 \%$ of patients with the highest HRU was nearly six times higher for patients with HCC-related endstage liver disease compared with 2.57 for those with non-HCC-related end-stage liver disease. Collectively, these results suggest that $\mathrm{CHC}$ complications could be better managed if treatment is initiated before disease has progressed to cirrhosis and emphasize the economic importance of initiating $\mathrm{CHC}$ treatment early.

While several studies have previously investigated the impact of the timing of $\mathrm{CHC}$ treatment initiation $[4,26,27]$, the economic burden of delaying antiviral treatment for $\mathrm{CHC}$ in Japan remained largely unknown. The results from the current analysis are consistent with the existing literature outside of Japan. Reau et al. [4] reported that the medical service costs incurred by treated patients were significantly lower than those incurred by untreated patients in the US, with up to $51 \%$ of the economic benefit attributable to EHM-related savings. Two modeling studies in the US assessed the health outcomes and costs of CHC patients at different stages of fibrosis $[26,27]$. Both studies concluded that early treatment of $\mathrm{CHC}$ led to higher quality-adjusted life-years and was costeffective. Taken together, the present findings and those from previous studies are consistent with the Japanese Society of Hepatology guidelines, which recommend that antiviral therapy be considered in all patients with $\mathrm{HCV}$ except those with decompensated cirrhosis or co-occurring conditions making them ineligible for treatment [10]. The current real-world study also confirms that costs can be reduced by initiating treatment early to decrease EHM- and HM-associated HRU [26, 27].

This study is subject to a number of limitations. First, the database may contain omissions and billing inaccuracies. Second, MDV data only covered acute care hospitals, which might have led to the inclusion of older and more vulnerable patients and the exclusion of patients with chronic conditions treated in non-acute care facilities. Although Japanese CHC patients are treated by hepatitis specialists instead of general practitioners [28], this may affect the generalizability of the results to the overall Japanese population. Third, the current study used diagnosis of cirrhosis as an indicator of delayed treatment initiation using a previously validated algorithm that uses claims data [21]. Different definitions of early versus delayed treatment initiation could potentially affect results. Fourth, medical claims could be associated with both hepatic and extrahepatic costs, which were identified based on diagnosis 
codes; however, this would not impact our estimation of the overall healthcare burden. Fifth, treated patients may or may not have sustained virologic response, which may lead to differences in costs and HRU between these two populations, but this information was not available in the database. Sixth, the pharmacy costs of treated $\mathrm{CHC}$ patients may have been overestimated because of price cuts of DAAs that occurred after the end of the data availability period in 2016, while medical costs associated with HMs and EHMs are expected to be relatively stable over time. This means the fraction of CHC-related pharmacy costs that is offset by savings in medical service costs may be even more important as of today. Finally, the presence of unobserved confounders cannot be excluded.

\section{CONCLUSION}

The current literature about the economic and healthcare impacts of withholding or delaying CHC treatment in Japan is scarce. This study, which was conducted in Japan, demonstrated that $\mathrm{CHC}$ treatment results in lower inpatient visits, which leads to a significant reduction in medical service costs, especially in early stages of liver disease. The specific reasons behind the higher HRU and medical service costs for untreated $\mathrm{CHC}$ patients and those receiving delayed treatments may warrant further research.

\section{ACKNOWLEDGEMENTS}

Funding. This study and the article processing charges were funded by AbbVie. All authors had full access to all of the data in this study and take complete responsibility for the integrity of the data and accuracy of the data analysis.

Authorship. All named authors meet the International Committee of Medical Journal Editors (ICMJE) criteria for authorship for this article, take responsbility for the integrity of the work as a whole, and have given their approval for this version to be published.

Medical Writing, Editorial, and Other Assistance. Medical writing assistance was provided by Samuel Rochette from Analysis Group, Inc. Support for this assistance was funded by Analysis Group, Inc., which received consultancy fees from AbbVie. The authors would like to thank Cheryl Q. Xiang and Olivia X. Liu from Analysis Group for their contribution related to analytical support. Financial support for these services was provided by AbbVie.

Disclosures. The design and conduct of the present study was approved by AbbVie, Inc., which participated in the interpretation of the data, the review, and the approval of the abstract. All authors contributed to the development of the publication and maintained control over the final content. Dendy Macaulay is an employee of Analysis Group, Inc., which received consultancy fees from AbbVie, Inc., to conduct this study. Yan Song is an employee of Analysis Group, Inc., which received consultancy fees from AbbVie, Inc., to conduct this study. Kiyotaka Yamazaki was an employee of AbbVie Japan at the time the study was conducted and may have owned stocks and/or stock options of the company. This author's current affiliation is Novartis Pharma K.K., Tokyo, Japan. Yuri Sanchez Gonzalez is an employee of AbbVie, Inc., and may own stocks and/or stock options.

Compliance with Ethics Guidelines. This article is based on previously available and deidentified data and does not involve any new studies of human or animal subjects performed by any of the authors. Appropriate permission from Medical Data Vision (MDV), the data provider, was obtained before using and analyzing the data.

Data Availability. The data sets generated and/or analyzed during the current study are not publicly available because of protections around the public sharing of private health information. 
Open Access. This article is distributed under the terms of the Creative Commons Attribution-NonCommercial 4.0 International License (http://creativecommons.org/licenses/ by-nc/4.0/), which permits any noncommercial use, distribution, and reproduction in any medium, provided you give appropriate credit to the original author(s) and the source, provide a link to the Creative Commons license, and indicate if changes were made.

\section{REFERENCES}

1. Burstow NJ, Mohamed Z, Gomaa AI, et al. Hepatitis $\mathrm{C}$ treatment: where are we now? Int J Gen Med. 2017;10:39-52.

2. Manns MP, Buti M, Gane E, et al. Hepatitis C virus infection. Nat Rev Dis Primers. 2017;3:17006.

3. Zampino R, Marrone A, Restivo L, et al. Chronic HCV infection and inflammation: clinical impact on hepatic and extra-hepatic manifestations. World J Hepatol. 2013;5(10):528-40.

4. Reau N, Vekeman F, Wu E, Bao Y, Gonzalez YS. Prevalence and economic burden of extrahepatic manifestations of hepatitis $\mathrm{C}$ virus are underestimated but can be improved with therapy. Hepatology Commun. 2017;1(5):439-52.

5. Franciscus A. Hepatitis C in Japan. 2015. http:// hcvadvocate.org/hepatitis/factsheets_pdf/HCAW_ Japan.pdf. Accessed 4 Sept 2017.

6. Yatsuhashi H. Past, present, and future of viral hepatitis C in Japan. Euroasian J Hepato-Gastroenterol. 2016;6(1):49-51.

7. Gower E, Estes C, Blach S, Razavi-Shearer K, Razavi H. Global epidemiology and genotype distribution of the hepatitis $\mathrm{C}$ virus infection. J Hepatol. 2014;61(1 Suppl):S45-57.

8. Petruzziello A, Marigliano S, Loquercio G, Cozzolino A, Cacciapuoti C. Global epidemiology of hepatitis $C$ virus infection: An up-date of the distribution and circulation of hepatitis $\mathrm{C}$ virus genotypes. World J Gastroenterol. 2016;22(34):7824-40.

9. Moriya T, Koyama T, Tanaka J, Mishiro S, Yoshizawa H. Epidemiology of hepatitis $C$ virus in Japan. Intervirology. 1999;42(2-3):153-8.

10. Asahina Y, Izumi N, Hiromitsu K, et al. JSH guidelines for the management of hepatitis $\mathrm{C}$ virus infection: a 2016 update for genotype 1 and 2 . Hepatol Res. 2016;46(2):129-65.

11. Ciesek S, Manns MP. Hepatitis in 2010: the dawn of a new era in HCV therapy. Nat Rev Gastroenterol Hepatol. 2011;8(2):69-71.

12. Hazuda DJ, Burroughs $M$, Howe AY, Wahl J, Venkatraman S. Development of boceprevir: a firstin-class direct antiviral treatment for chronic hepatitis C infection. Ann N Y Acad Sci. 2013;1291:69-76.

13. Crespo J, Cabezas J, Sacristan B, et al. Barriers to HCV treatment in the era of triple therapy: a prospective multi-centred study in clinical practice. Liver Int. 2015;35(2):401-8.

14. King A, Bornschlegel K, Johnson N, Rude E, Laraque F. Barriers to treatment among New York City residents with chronic hepatitis C virus infection, 2014. Public Health Rep. 2016;131(3):430-7.

15. Lo Re V III, Gowda C, Urick PN, et al. Disparities in absolute denial of modern hepatitis $C$ therapy by type of insurance. Clin Gastroenterol Hepatol. 2016;14(7):1035-43.

16. Schaeffer S, Khalili M. Reasons for HCV non-treatment in underserved African Americans: implications for treatment with new therapeutics. Ann Hepatol. 2015;14(2):234-42.

17. Virabhak S, Yasui K, Yamazaki K, et al. Cost-effectiveness of direct-acting antiviral regimen ombitasvir/paritaprevir/ritonavir in treatmentnaive and treatment-experienced patients infected with chronic hepatitis $C$ virus genotype $1 \mathrm{~b}$ in Japan. J Med Econ. 2016;19(12):1144-56.

18. Lauffenburger JC, Mayer CL, Hawke RL, Brouwer KL, Fried MW, Farley JF. Medication use and medical comorbidity in patients with chronic hepatitis C from a US commercial claims database: high utilization of drugs with interaction potential. Eur J Gastroenterol Hepatol. 2014;26(10):1073-82.

19. Saeed MJ, Olsen MA, Powderly WG, Presti RM. Diabetes mellitus is associated with higher risk of developing decompensated cirrhosis in chronic hepatitis $\mathrm{C}$ patients. J Clin Gastroenterol. 2017;51(1):70-6.

20. LaMori J, Tandon N, Laliberte F, et al. Predictors of high healthcare resource utilization and liver disease progression among patients with chronic hepatitis C. J Med Econ. 2016;19(4):364-73.

21. Nehra MS, Ma Y, Clark C, Amarasingham R, Rockey DC, Singal AG. Use of administrative claims data for identifying patients with cirrhosis. J Clin Gastroenterol. 2013;47(5):e50-4. 
22. National Comprehensive Cancer Network. NCCN Clinical Practice Guidelines in Oncology-Melanoma Version 2.2018. 2018. https://www.nccn. org/professionals/physician_gls/pdf/melanoma.pdf.

23. Charlson ME, Pompei P, Ales KL, MacKenzie CR. A new method of classifying prognostic comorbidity in longitudinal studies: development and validation. J Chronic Dis. 1987;40(5):373-83.

24. Quan H, Li B, Couris CM, et al. Updating and validating the Charlson comorbidity index and score for risk adjustment in hospital discharge abstracts using data from 6 countries. Am J Epidemiol. 2011;173(6):676-82.

25. Berger ML, Sox H, Willke RJ, et al. Good practices for real-world data studies of treatment and/or comparative effectiveness: recommendations from the joint ISPOR-ISPE Special Task Force on real- world evidence in health care decision making. Pharmacoepidemiol Drug Saf. 2017;26(9):1033-9.

26. Chahal HS, Marseille EA, Tice JA, et al. Cost-effectiveness of early treatment of hepatitis $\mathrm{C}$ virus genotype 1 by stage of liver fibrosis in a US treatment-naive population. JAMA Intern Med. 2016;176(1):65-73.

27. Leidner AJ, Chesson HW, Xu F, Ward JW, Spradling PR, Holmberg SD. Cost-effectiveness of hepatitis C treatment for patients in early stages of liver disease. Hepatology. 2015;61(6):1860-9.

28. Oza N, Isoda H, Ono T, Kanto T. Current activities and future directions of comprehensive hepatitis control measures in Japan: the supportive role of the Hepatitis Information Center in building a solid foundation. Hepatol Res. 2017;47(6):487-96. 